\title{
Kisspeptin and RFRP3 modulate body mass in the Phodopus Sungorus via two different neuroendocrine pathways
}

\author{
Fernando Cázarez-Márquez ${ }^{1,2,3}$, Sebastien Milesi ${ }^{1}$, Marie-Pierre Laran-Chich ${ }^{1}$, Paul Klosen ${ }^{1}$, Andries \\ Kalsbeek $^{2,3,4}$, Valérie Simonneaux ${ }^{1}$ \\ ${ }^{1}$ Institute of Cellular and Integrative Neurosciences (INCI), Strasbourg, France \\ ${ }^{2}$ Netherlands Institute for Neuroscience (NIN), Amsterdam, The Netherlands \\ ${ }^{3}$ Laboratory of Endocrinology, Amsterdam UMC, University of Amsterdam, Amsterdam \\ Gastroenterology \& Metabolism, Amsterdam, The Netherlands \\ ${ }^{4}$ Department of Endocrinology and Metabolism, Amsterdam UMC, University of Amsterdam, \\ Amsterdam, the Netherlands
}

Corresponding author: Valérie Simonneaux, Institute of Cellular and Integrative Neurosciences (INCI), Strasbourg, France, email: simonneaux@inci-cnrs.unistra.fr

Short title: RFamide control of seasonal changes in body weight

Keywords: Kisspeptin, RFRP3, Reproduction, Energy metabolism, Phodopus sungorus, Neuroendocrinology 
2 Many animals exhibit remarkable metabolic and reproductive adaptations to seasonal changes in their

3 environment. When day length shortens, Djungarian hamsters (Phodopus sungorus) reduce their body 4 weight and inhibit their reproductive activity, while the opposite occurs in springtime. These physiological 5 adaptations are thought to depend on photoperiodic changes in hypothalamic genes encoding the 6 peptides kisspeptin and RFRP3 for the control of reproduction, and pro-opiomelanocortin and 7 somatostatin for metabolic regulation. The objective of this study was to investigate the effect of 8 kisspeptin and RFRP3 on long-term body weight regulation in order to establish whether metabolic and 9 reproductive hypothalamic networks may interact during adaptation to seasonal physiology. We found 10 that chronic central administration of both kisspeptin or RFRP3 in short photoperiod-adapted male 11 Djungarian hamsters increased body weight although through different pathways. The effect of kisspeptin 12 was dependent on testicular activity, as castration prevented the body weight increase, and was 13 associated with an increase in pro-opiomelanocortin and neuropeptide $\mathrm{Y}$ expression. On the other hand, 14 the orexigenic effect of RFRP3 was associated with an increase in circulating insulin and leptin levels, but 15 had no effect on any of the hypothalamic metabolic genes investigated, and did not change circulating 16 levels of sex steroids. Notably, neither kisspeptin nor RFRP3 altered female hamster's metabolic 17 parameters. Thus, using a rodent model exhibiting seasonal changes in reproduction and metabolism, this 18 study demonstrates that in addition to their role in the central control of reproduction, kisspeptin and 19 RFRP3 also participate in body weight control in a sex-dependent manner, kisspeptin's effects involving 20 the anabolic action of testosterone and RFRP3 acting on adiposity. 
23 Mammals adapt their metabolic and reproductive activities to the seasonal changes in the environment

24 to ensure that offspring will be born in favorable conditions with enough resources for survival. Seasonal

25 rodents like Djungarian hamsters (Phodopus sungorus) increase their food intake and body weight and

26 activate their reproduction with increasing day length in spring and summer, whereas the opposite occurs

27 when days are shortening in autumn and winter ${ }^{1-3}$. These annual changes in metabolism and reproduction

28 are driven by the pineal hormone melatonin, whose nocturnal production depends on the length of the

29 night ${ }^{4}$.

30 In the last ten years, major progress has been made to uncover the melatonin-regulated neuronal

31 networks that control seasonal reproduction and metabolism. Two hypothalamic RF-amide peptides,

32 kisspeptin (Kp) and RFamide related peptide 3 (RFRP3), both known to regulate GnRH neuronal activity

33 and gonadotropin secretion ${ }^{5,6}$, display marked melatonin-driven seasonal variation in a number of

34 seasonal species, including rodents ${ }^{7-10}$. Notably, in all seasonal species investigated so far, RFRP3

35 expression is markedly inhibited in short day (SD) conditions, whereas the photoperiodic regulation of Kp

36 expression is modulated by the feedback of sex steroids ( ${ }^{11,12}$ for reviews). Further, chronic central

37 infusions of Kp or RFRP3 in SD-adapted Syrian hamsters have been shown to restore reproductive activity

38 despite the photo-inhibitory conditions ${ }^{7,13,14}$. Also, changes in hypothalamic genes encoding peptides

39 related to energy homeostasis, such as pro-opiomelanocortin (Pomc) and somatostatin (Sst), have been

40 associated with the SD-induced metabolic reduction observed in Djungarian hamsters ${ }^{15,16}$.

41 Previous observations in non-seasonal rodents, suggest that Kp and RFRP3 may as well regulate metabolic

42 activity. Disruption of the Kp receptor in mice not only interferes with reproductive activity, but also with

43 the control of body weight and Pomc mRNA expression ${ }^{17,18}$. Similarly, mutation of the receptor for RFRP3

44 alters the metabolic response to fasting conditions in mice ${ }^{19}$ and a central injection of RFRP3 increased 
45 food intake in rats as well as Npy mRNA ${ }^{20,21}$. Therefore, the objective of this study was to investigate 46 whether the seasonal changes in Kp and RFRP may impact the seasonal control of body weight. We 47 assessed whether chronic central administration of Kp or RFRP3 in SD-adapted male and female 48 Djungarian hamsters would restore the long day (LD)-related metabolic phenotype, and aimed to identify 49 the putative hypothalamic targets involved.

\section{Materials and methods}

\section{Animals}

53 Post-pubertal (three to four months old at the beginning of experiments) male ( $n=72)$ and female ( $n=63)$

54 Djungarian hamsters were used. The animals were born and raised in the Chronobiotron animal unit (UMS 553415 , Strasbourg, France). They were housed individually with an enriched environment known to reduce 56 the depression like behavior caused by isolation, with food (Altromin 720, GmbH \& Co, Lage, Germany) 57 and water ad libitum, and under long day conditions (LD: 16h light - 8h darkness) unless otherwise stated.

58 All the manipulations and experiments were done in accordance with the local ethical committee 59 (CREMEAS) and the French National Ministry of Education and Research (authorization \# 60 2015021010234017).

61 Experimental design

62 RFRP3 (TLSRVPSLPQRFa Caslo, Denmark) and Kp10, the shortest active form of Kp (YNWNSFGLRYa; Caslo,

63 Denmark), were dissolved in artificial cerebral fluid (aCSF) and placed in osmotic minipumps (Alzet ${ }^{\circledR}$ Model 64 1004). The $\mathrm{Kp}$ dose of $170 \mathrm{pmol} /$ hour $(2 \mathrm{mg} / \mathrm{mL})$ was selected from a previous experiment ${ }^{14}$ and maximal 65 solubility reported by the manufacturer (ToCris Bioscience). RFRP3 is known to show clear dose-dependent 66 effects ${ }^{8,13}$. Here, a dose of $8.25 \mathrm{pmol} /$ hour $(105 \mu \mathrm{g} / \mathrm{mL})$ was selected from a pilot experiment with doses 
67 ranging from $2.75 \mathrm{\rho mol} /$ hour to $250 \mathrm{pmol} / \mathrm{hour}$ in both male $(n=24)$ and female $(n=38)$ animals (Figure 68 1).

69 Experiment 1.The aim of this experiment was to test the effects of intracerebroventricular (ICV) applied 70 Kp10 or RFRP3 on body weight and food intake in male and female Djungarian hamsters. Seventeen male 71 and nineteen female animals were transferred for 10 weeks to short-day conditions (SD: 8h light - 16h 72 darkness) in order to inhibit reproduction and cause a reduction in food intake and body weight. In the $7310^{\text {th }}$ week, animals, exhibiting the expected reproductive inhibition and body weight loss, were separated 74 randomly into three groups (SD + Kp10, SD + RFRP3, SD + aCSF, $n=5-7$ of each sex per group) and 75 implanted with the corresponding osmotic minipumps for 5 weeks. In parallel, five male and six female 76 animals, kept in LD during the whole experiment, were implanted with an aCSF filled osmotic pump (LD + 77 aCSF) at the same time as the SD adapted animals to serve as control animals and reference for an active 78 reproduction and high metabolic activity (no body weight loss).

79 Experiment 2. The objective of this experiment was to test the sex steroid dependency of the Kp10 effects 80 observed in Experiment 1. Twenty-six male Djungarian hamsters were transferred to the SD conditions as 81 described before. In the $10^{\text {th }}$ week, the animals were gonadectomized (SD Gx, n=13) or sham operated (SD, $82 \mathrm{n}=13$ ) and implanted with osmotic minipumps containing either Kp10 (170 pmol/hour) or aCSF for 5 weeks 83 and assigned to either one of 4 groups (1: SD + Kp10; 2: SD + aCSF; 3: SD Gx + aCSF; 4: SD Gx + Kp10; $n=6$ $84 \quad-7$ animals per group).

85 For both experiments, body weight and food intake were measured manually with a weighing machine 86 once a week during 5 weeks. After 5 weeks, animals were sacrificed by $\mathrm{CO}_{2}$ inhalation, blood was collected

87 by a heart puncture and animals were perfused transcardially with phosphate-buffered saline (PBS) 88 followed by paraformaldehyde-lysine-periodate (PLP) fixative and embedded in polyethylene glycol as 89 described before ${ }^{22}$. After 15 weeks of treatment none of SD + aCSF male or female hamsters showed 
90 refractoriness that is known to occur spontaneously from the 16-20 week of SD-adaptation. Blood-heparin

91 samples were centrifuged and plasma was stored at $-20^{\circ} \mathrm{C}$ for hormone measurements. All the animals

92 were sacrificed in ad libitum conditions during the light (sleep) phase.

93 Surgery

94 Minipump implantation was performed as described previously for the Djungarian hamster ${ }^{14}$. Briefly,

95 minipumps were prepared 72 hours before implantation and maintained at $37^{\circ} \mathrm{C}$ until surgery according

96 to the manufacturer instructions. Animals were anesthetized with Rompun (Bayer Pharma, Puteaux,

97 France) and Zoletil (Virbac, Carros, France) and the L-shaped cannula of the brain kit connected to the

98 minipump was implanted in the lateral ventricle (coordinates: $1.5 \mathrm{~mm}$ lateral to bregma, $2.5 \mathrm{~mm}$ ventral

99 to the dura), while the body of the pump was placed subcutaneously at the back of the animal.

100 For experiment 2, just before the minipump implantation and after anesthesia, thirteen hamsters were

101 castrated by a one-centimeter laparotomy exposing the epidydimal white adipose tissue (eWAT) to quickly

102 localize and dissect the testes. The remaining animals were sham-operated meaning that eWAT was

103 exposed but gonads were kept intact.

104 Non radioactive in situ hybridization

105 Polyethylene glycol-embedded brains were sectioned from the preoptic area to the mammillary bodies 106 into serial $10 \mu \mathrm{m}$ thick sections using a microtome. Series of sections were mounted selecting one out of 107 every 12th section (the distance between two sections being $110 \mu \mathrm{m}$ ). Sections were mounted on 108 Superfrost Plus ${ }^{\mathrm{TM}}$ slides and stored at $-80^{\circ} \mathrm{C}$ until use. Non-radioactive in situ hybridization using 109 digoxygenin labeled riboprobes was performed as reported before ${ }^{23,24}$. The rat neuropeptide $Y$ (Npy) 110 probe (87-522 of Genbank NM_012614.2; 91\% of homology with the hamster's sequence), rat Pomc probe 111 (157-731 of Genbank NM_139326, sequence verified by restriction mapping) and rat Sst probe (135-465 
112 of Genbank NM_012659.2, 92-93\% homology with the hamster's sequence) were transcribed from a 113 linearized plasmid in the presence of digoxigenin-labeled nucleotides (Roche, Meylan France).

114 The sections were post-fixed with $4 \%$ formaldehyde, digested during 30 minutes at $37^{\circ} \mathrm{C}$ with $0.5 \mu \mathrm{g} / \mathrm{mL}$ 115 proteinase K (Roche, Meylan France) and acetylated twice for 10 minutes in $100 \mathrm{mM}$ triethanolamine and $1160.25 \%$ acetic anhydride. The slides were hybridized with $400 \mathrm{ng} / \mathrm{mL}$ of labelled antisense probe in $50 \%$ 117 formamide, $5 X$ SSC, $5 X$ Denhardt's solution and $1 \mathrm{mg} / \mathrm{mL}$ Salmon sperm DNA for $38-40 \mathrm{~h}$ at $60^{\circ} \mathrm{C}$. A high 118 temperature stringency wash with $0.1 \times \mathrm{SSC}$ at $72^{\circ} \mathrm{C}$ was applied $6 \times 10$ minutes to eliminate all the non119 hybridized probes. The digoxigenin tag was detected using an alkaline phosphatase-coupled anti120 digoxigenin antibody (1:5000, Roche, Meylan, France). Alkaline phosphatase activity was visualized with a

121 mixture of Nitro Blue Tetrazolium/Bromo-Chloro-Indolyl Phosphate for one to two hours and stopped 122 before the staining intensity reached saturation. Hybridization with corresponding sense probes gave no 123 signal, indicating specificity of the antisense probes.

$124 \quad$ Hormone measurements

125 Leptin levels were measured in one time diluted plasma samples using the Multi Species RIA Leptin Kit 126 (Millipore, \# XL-85K, France) according to the manufacturer's protocol. Intra-assay variations was 3.4 to $1273.6 \%$ and inter-assay variation 6.5 to $8.7 \%$. The sensitivity of the assay was $0.8 \mathrm{ng} / \mathrm{mL}$.

128 Insulin was measured in 20 times diluted plasma samples using the Hamster Insulin ELISA kit (Crystal Chem $129 \# 90336$, USA). Intra and inter-assay variations were less than $10 \%$. The limit of sensitivity of the assay was $130 \quad 0.1 \mathrm{ng} / \mathrm{mL}$.

131 Blood glucose levels were determined directly at the time of the sacrifice with the glucometer Accu-Check 132 Performa Test strips ${ }^{\circledast}$ (Roche Diagnostic, France). The range of values for the measurements was from 1.81 133 to $600 \mathrm{mg} / \mathrm{dL}$. 
134 Testosterone was measured in 10 times diluted plasma samples using an ELISA kit from R\&D Systems 135 (\#KGE010, Minneapolis, USA) according to the manufacturer protocol. The intra-assay variation was $2.9 \%$ 136 to $4 \%$ and the inter-assay variation $5.6 \%$ to $6.8 \%$. The sensitivity of the assay was $0.030 \mathrm{ng} / \mathrm{mL}$.

Image Analysis

139 A person unaware of the animal's experimental set up quantified the in situ hybridization signals. A CCD 140 camera (Sony Model 77CE) attached to a microscope (Zeiss Axios-kop with Plan-NEOFLUAR Zeiss 141 objectives, Carl Zeiss GmbH, Germany) was used to take micrographs with an $10 \times 0.63$ objective. For each 142 gene analyzed, pictures of the area of interest were taken at the same time with identical lighting 143 conditions for all animals. For each animal, the total number of cells showing labeling of a given mRNA was 144 counted manually in all sections of the area of interest, and given as number of mRNA positive neurons 145 per animal, as reported previously ${ }^{14,25}$. The number of neurons expressing Npy or Pomc are the total 146 amount of neurons counted in the entire series of sections stained. SST neuron numbers were counted 147 only in the caudal part of the arcuate nucleus (ARC) (Bregma -2.3 to -2.54 ) as SST expression shows 148 photoperiodic variation only at this level ${ }^{16,23}$. The mean in situ hybridization signal intensity per neuron 149 was analyzed with Image J software using a fixed sized circle for each analyzed neuron. Analysis was 150 performed in anterior, medial and posterior (Npy or Pomc) or only in posterior (Sst) ARC sections for each 151 animal. Labeling intensity was measured in 30 to 40 neurons per section quantified (i.e., 90 or 120 neurons 152 per animal), based on previous work demonstrating that this number of cells provides a stable mean 153 intensity ${ }^{23}$.

154 Statistical Analysis

155 All data are presented as mean \pm SEM. All the graphs and statistical analyses were done using GraphPad 156 Prism Software Inc. Statistical significance was set at $p<0.05$. Body weight, food intake data after 5 weeks 
treatments with RFamides, hormone assays and in situ hybridization were analyzed with One-way ANOVA and the Tukey post-hoc honestly significant difference (HSD) test. Body weight, food intake and seminal vesicles from experiment 2 have been analyzed by Two-way ANOVA and Bonferroni post-hoc HSD.

Results

\section{Differential reproductive effects of chronic Kp10 or RFRP3 in SD-adapted hamsters}

As expected, adaption to SD induced a marked decrease in the weight of male and female reproductive organs (Fig. 2). Five weeks ICV administration of Kp10 in SD-adapted hamsters increased the weight of the testes $(F(3,18)=74.7 ; P<0.0001)$, seminal vesicles $(F(3,18)=130.2 ; P<0.0001)$ and uteri $(F(3,21)=12.36$; $\mathrm{P}<0.0001$ ) up to the values observed in LD-adapted hamsters (Fig. 2). By contrast, administration of different doses of RFRP3 (from 2.75 to 250 pmol/hour) did not increase the size of the reproductive organs

168 in either sex (Fig. 2). In male hamsters, the measure of circulating testosterone confirmed the differential reproductive effect of Kp10 $(39.51 \pm 7.8 \mathrm{ng} / \mathrm{mL})$ and RFRP3 $(0.64 \pm 0.01 \mathrm{ng} / \mathrm{mL}$, i.e., not different from aCSF treatment, $0.5 \pm 0.03 \mathrm{ng} / \mathrm{mL})$ in SD-adapted animals $(F(4,19)=21.34, \mathrm{P}<0.0001)$.

\section{Sex-dependent effects of chronic Kp10 or RFRP3 on body weight, food intake, and metabolic} hormones in SD-adapted hamsters

173 Ten weeks after transfer to the SD photoperiod, hamsters displayed a lower body weight $(33.30 \pm 1.85 \mathrm{~g}$ 174 for male; $29.55 \pm 1.68 \mathrm{~g}$ for female) as compared to LD conditions ( $46.52 \pm 1.85 \mathrm{~g}$ for male; $38.61 \pm 1.45 \mathrm{~g}$ 175 for female) (Fig. 3A, B). In SD-adapted male hamsters, chronic treatment with either 170 pmol/hour Kp or $1768.25 \mathrm{pmol} / \mathrm{hour}$ RFRP3 for 5 weeks increased body weight as compared to aCSF control animals that 177 showed no weight gain $(F(3,18)=13.03 ; P=0.0001)$ (Fig. $3 A)$. The effect of RFRP3 treatment, but not that 178 of Kp10, was associated with a significant increase in the weekly food intake when compared to aCSF 
control animals (ICV Treatment: $\mathrm{F}(3,18)=10.84$, P=0.0003; Time: $\mathrm{F}(4,72)=3.821$, $\mathrm{P}=0.0071$; Interaction

$180 \mathrm{~F}(12,72)=1.385, \mathrm{P}=0.1929)$ as well as the cumulative food intake along the duration of the treatment 181 when compared to aCSF control animals $(F(3,18)=7.3 ; P=0.020)$ (Fig. $3 A)$. Of note, none of the other 182 doses of RFRP3 tested induced a significant change in the male's body weight (Fig. 1A). In SD-adapted 183 female hamsters, none of the peptides altered body weight $(F(3,21)=1.898 ; P=0.1609)$ or food intake

184 (Treatment: $F(2,15)=1.044 ; P=0.3763$; Time: $F(4,60)=1.675 ; P=0.1677 ;$ Interaction: $F(8,60)=0.6686$; $185 \quad \mathrm{P}=0.7169)$ ) (Fig. 3B).

186 In both male and female hamsters, circulating insulin and leptin, but not glucose, concentrations were 187 significantly lower in SD- as compared to LD-adapted animals (Fig. 3). In SD-adapted male hamsters, RFRP3 treatment increased circulating insulin $(F(2,10)=7,977 ; P=0.0085$; Tukey HSD P=0.0126) and leptin $(F(2$, 13) $=13.78 ; P=0.0002 ;$ Tukey HSD $P=0.0014$ ) levels as compared to aCSF treated animals, but had no effect on glucose $(F(2,14)=0.02254 ; P=0.9778$; Tukey HSD $P=0.9858)(F i g .3 A)$. On the other hand, Kp10 treatment in SD male hamsters had no effect on glucose, insulin or leptin (Fig. 3A). In SD-adapted female hamsters, neither RFRP3 nor Kp10 altered the circulating levels of glucose $(F(2,16)=0.8513 ; P=0.5498)$, insulin $(F(2,16)=0.7942 ; P=0.4690)$ or leptin $(F(2,14)=4.196 ; P=0.0373)$ as compared to the $S D$ control group (Fig. 3B). All these measurements come from animals with ad libitum access to food and water.

\section{Effects of chronic Kp10 or RFRP3 on expression of metabolic genes in the hypothalamus of SD-} adapted hamsters

In order to find putative hypothalamic targets of the Kp10 and RFRP3 treatment, the number of neurons expressing the metabolic genes Npy, Pomc and Sst and the intensity of the mRNA labeling per neuron were quantified in the ARC at the end of the 5-week ICV treatments (Figs. 4-5). 

was lower in LD- as compared to SD-adapted male (Fig. 4C) and female (Fig. 5C) hamsters. No 203 photoperiodic variation was observed in the number of NPY neurons or in the neuronal Npy mRNA 204 intensity signal in male (Fig. 4B) or female (Fig. 5B) hamsters.

In SD-adapted male hamsters, chronic Kp10 treatment increased the number of Pomc (Fig. 4A) $(\mathrm{F}(3,18)=$ 20.48; $\mathrm{P}<0.0001)$ and Npy (Fig. 4B) $(\mathrm{F}(3,17)=4.25 ; \mathrm{P}=0.0206)$ expressing neurons and did not change Sst expression (Fig. 4C). Chronic RFRP3 treatment by contrast had no effect on the expression of the investigated genes in the male hamsters (Fig. 4). In SD-adapted female hamsters, neither Kp10 nor RFRP3 treatment altered the number or intensity of neurons expressing the investigated genes (Fig. 5).

\section{4. Sex steroid dependence of Kp10 effect in the male hamsters}

211 In order to investigate whether the effect of Kp10 on body weight in male hamsters was sex steroid 212 dependent, in Experiment 2 we repeated the Kp10 infusion in castrated (Gx) SD-adapted animals. As 213 expected, Gx blocked the Kp10 induced increase in seminal vesicle weight (Castration: $F(1,21)=107.5$, $214 \mathrm{P}<0.0001$; ICV treatment: $\mathrm{F}(1,21)=118.5, \mathrm{P}<0.0001$; Interaction: $\mathrm{F}(1,21)=110.8, \mathrm{P}<0.0001)$ (Fig. $6 \mathrm{~A})$ 215 confirming the absence of circulating testosterone. Further, the Kp10-induced increase in body weight was 216 totally abolished by $\mathrm{Gx}($ Castration: $\mathrm{F}(1,21)=4.884$, $\mathrm{P}=0.0383$; ICV treatment: $\mathrm{F}(1,21)=18.05 ; \mathrm{P}=0.0004$;

217 Interaction: $F(1,21)=7.666 ; P=0.0115$ ) (Fig. 6B). Finally, $G x$ had no significant effect on the cumulative 218 food intake and Kp10 did not modify food intake in either sham or Gx male hamsters (Castration: F (1, 21) $219=0.03778, P=0.8477 ;$ ICV treatment: $F(1,21)=4.232 ; P=0.0523 ;$ Interaction: $F(1,21)=0.1564 ; P=0.6964)$ 220 (Fig. 6C). 
224 Different groups have investigated the hypothalamic mechanisms involved in seasonal metabolism and 225 reproduction, highlighting the roles of POMC, SST, Kp, and RFRP3 neurons in either one or both of these 226 processes ${ }^{14,26-28}$. In the present study, we investigated putative central mechanisms responsible for the 227 seasonal synchronization between body weight control and reproduction, including possible sex228 differences. Notably, we observed sex-dependent and differential effects of central Kp and RFRP3 on body 229 weight and metabolic hormones in the seasonal Djungarian hamster. Both Kp and RFRP3 increased body 230 weight in SD-adapted male, but not female, hamsters. The effect of $\mathrm{Kp}$, was testosterone-dependent and 231 involved changes in Npy and Pomc gene expression, whereas the effect of RFRP3, but not Kp, was 232 accompanied by an increase in food intake and circulating levels of insulin and leptin.

\section{Sex-dependent central effect of RF-amides on body weight}

234 Male and female Djungarian hamsters displayed the expected marked reduction in body weight and 235 reproduction when exposed to SD photoperiodic conditions. The decrease in body weight correlated with 236 a reduction in food intake and a down-regulation of plasma insulin and leptin levels as reported previously 23729,30 . In the hypothalamus, we confirmed the SD-induced down regulation of Pomc gene expression in the 238 ARC of both sexes ${ }^{15,31}$ and found that the previously reported SD-induced up regulation of Sst gene 239 expression in the posterior part of the male hamster ARC ${ }^{16,23}$ was also present in females. We also 240 confirmed the lack of SD-induced changes in Npy expression as previously described ${ }^{15,31}$.

241 Importantly, we demonstrated that a 5-week central administration of either Kp or RFRP3 increased body 242 weight in SD-inhibited lean male Djungarian hamsters. However, neither Kp nor RFRP3 were observed to 243 modulate female hamsters' body weight and food intake, although the Kp treatment did restore female 244 reproductive activity as attested by uterus weight. These data indicate that the metabolic effects of central $245 \mathrm{Kp}$ and RFRP3 are sex-dependent, despite the seasonal changes in metabolic Pomc and Sst ${ }^{15,31}$ and 
reproductive Kiss1 and Rfrp ${ }^{14,32}$ gene expression being similar among male and female Djungarian

247 hamsters, as also observed in the present study. The orexigenic effect of central RFRP3 has already been 248 reported in several mammalian species ${ }^{20,21,25}$, whereas for $\mathrm{Kp}$ an acute anorexigenic effect in fasted female

249 jerboas $^{25}$ and a lack of effect in either ad libitum or fasted rats has been reported ${ }^{33}$. Although there is no 250 clear explanation as to why chronic Kp and RFRP3 do not alter female Djungarian hamster's body weight,

251 the sex difference in energy metabolism and obesity susceptibility is well known, including previous studies 252 reporting sex-dependent metabolic effects of the currently studied peptides. For instance, it was reported 253 that Kp receptor mutation in mice affects body weight and glucose metabolism of female but not male 254 mice ${ }^{17}$. The contrasting sex-dependent metabolic effect of $\mathrm{Kp}$ in mice and hamsters may rely on the 255 different species and/or experimental paradigms used in both studies, with a life-long kisspeptin signaling 256 deficiency in the knockout mice as compared to chronic kisspeptin treatment of several weeks in the 257 seasonal hamsters. Sensitivity to Kp may also be different between sexes, as for instance, in adult hamsters $258{ }^{34}$ and prepubertal rats ${ }^{35}$, acute kp10 injections produce smaller effect of gonadotropins secretion in 259 females than in males. In line with this observation, hypothalamic kisspeptin receptor expression has been 260 found to be lower in female than male stripped hamsters ${ }^{36}$.

\section{Two RF-amides, two pathways}

262 It has become clear that the hypothalamic Kp and RFRP3 neurons are a functional link between the 263 seasonal changes in the pineal hormone melatonin and reproduction ( ${ }^{37}$ for review). Thus, melatonin acts 264 on the pars tuberalis to control TSH production which, in turn, regulates deiodinase activity in tanycytes 265 located in the wall of the third ventricle leading to seasonal changes in the content of T3 in the mediobasal 266 hypothalamus. Remarkably, a central administration of $\mathrm{TSH}^{23}$ or $\mathrm{T3}^{38}$ can rescue the body weight reduction 267 and the inhibited reproduction of SD-adapted male hamsters, as well as the LD phenotype of Kp and RFRP3 268 expression ${ }^{23,39}$. 
269 Chronic infusion of $\mathrm{Kp}^{7}$ or RFRP3 ${ }^{13}$ has already been reported to rescue reproductive activity in SD270 adapted Syrian hamsters. Here, both peptides are also found to rescue body weight loss in SD-adapted 271 male Djungarian hamsters. Despite their similar effects on the body weight, the mode of action of Kp10 272 and RFRP3 differed in several aspects. First, the metabolic effect of Kp10 is probably mediated via an 273 increased activity of the hypothalamo-pituitary-gonadal axis, since castration completely abolished the 274 Kp10-induced increase in body weight. On the other hand, RFRP3 treatment increased animal's body 275 weight without a restoration of reproductive activity. The lack of reproductive effect of chronic RFRP3 in 276 the SD-inhibited Djungarian hamster contrasts with what has been observed in the Syrian hamster ${ }^{13}$. The 277 acute effect of RFRP3 on LH secretion in male Djungarian hamster depends on its photoperiodic status, 278 being stimulatory in the SD- and inhibitory in the LD-adapted animals ${ }^{8}$. Based on these findings, we 279 expected increased reproductive activity after chronic RFRP3 in the male Djungarian hamsters, although 280 there is also some evidence indicating that RFRP3 may inhibit reproduction in intermediate photoperiods 281 (13.5 h Light) ${ }^{40}$. Further, it is important to note that RFRP3 displays marked dose-dependent effects ${ }^{8,13}$ 282 and in the present study only a dose of $8.25 \mathrm{pmol} /$ hour was able to induce a metabolic effect among the 283 different RFRP3 concentrations tested. It is likely that such a marked dose-dependent effect of RFRP3 is 284 due to a change in receptor density, sensitivity or signaling. Previously it has been reported that the SD285 induced decrease in Djungarian hamster's body weight is both independent and dependent of the gonads $286{ }^{41}$, our data indicate that Kp10 may be involved in the testosterone-dependent and RFRP3 in the 287 testosterone-independent seasonal regulation of the male hamster's body weight.

288 Strikingly, we found that the RFRP3- but not the Kp10-treated males increased their food intake and 289 showed higher circulating leptin and insulin levels. Leptin provides an indirect measurement of the white 290 adipose tissue (WAT) content in the body, indicating that in RFRP3-treated animals the increased body 291 weight might be due primarily to increased adiposity. Although in our experiment we did not assess 292 directly the fat mass content, our results are supported by recent findings reporting increased WAT 
293 accumulation in mice injected intraperitoneally with RFRP3 ${ }^{42}$. It is well known that increased adiposity 294 results in insulin resistance ${ }^{43}$, therefore, the increased plasma insulin levels found in the RFRP3-treated 295 male hamsters might be due a compensatory mechanism to maintain stable glucose levels. Previously, 296 leptin and insulin signaling have been reported to be differentially modulated in LD versus SD-adapted 297 Djungarian hamsters ${ }^{44,45}$, indicating the Djungarian hamster as an interesting animal model to study both, 298 leptin and insulin sensitivity. Here our data indicate that photoperiodic changes in RFRP3 may possibly be 299 implicated in this phenomenon.

300 The ARC is pivotal for metabolic regulation and because recent studies have shown that the Kp receptor 301 Kiss1r ${ }^{46}$ and the RFRP3 receptor GPR147 ${ }^{47}$ are both expressed in this brain area, we analyzed whether 302 Kp10 or RFRP3 could target ARC metabolic genes. Chronic Kp10 increased Pomc and Npy in males, but not 303 in females, which could explain why only male hamsters modified their metabolic phenotype. POMC 304 neurons receive a dense $\mathrm{Kp}$ innervation and express Kiss $1 \mathrm{r}$ in rats ${ }^{48}$, they are directly activated by $\mathrm{Kp}$ in 305 mice ${ }^{49}$ and are regulated by a central Kp10 injection in jerboas ${ }^{25}$, altogether supporting a direct effect of $306 \mathrm{Kp}$ on POMC neurons. On the other hand, castration completely abolished the effect of Kp10 on body 307 weight and since testosterone is known to regulate Pomc ${ }^{50-52}$ and $\mathrm{Npy}^{53-55}$, probably also part of the Kp 308 effect on the metabolic genes is testosterone-dependent. The up-regulation of Pomc expressing neurons 309 in animals with increased body weight may seem counterintuitive, but this observation matches the higher 310 Pomc gene expression observed in the heavier LD-adapted hamsters ${ }^{15}$. In addition, even when the Pomc 311 gene is down-regulated in SD, the levels of $\alpha-\mathrm{MSH}$, an anorexigenic peptide produced by the Pomc gene 312 might be higher due to a reported increased activity of carboxipeptidase $E^{56,57}$. Therefore, in principle it is 313 possible that the Kp10-induced increase in Pomc expression is associated with lower $\alpha$-MSH. Further 314 research on the production of either $\alpha-\mathrm{MSH}$ or $\beta$-endorphin is necessary to conclude on the exact 315 molecular targets of Kp for the photoperiodic regulation of metabolism. In contrast to POMC, NPY/AgrP 316 neurons exhibit a very low level of Kiss $1 r^{48}$ and Kp10 has been reported to exert an indirect local inhibitory 
317 effect on these neurons ${ }^{49,58}$. Further, when a metabolic effect of Kp10 or Kiss1r mutation was reported in 318 female rodents, this effect was not associated with changes in Npy expression ${ }^{18,25}$. Therefore, we assume 319 that the increased number of NPY neurons in Kp10 treated male hamsters essentially depends on the 320 increased testosterone secretion following testis reactivation ${ }^{53-55}$.

321 The finding in males that the RFRP3-induced increase in body weight and circulating insulin and leptin 322 levels was not associated with changes in metabolic genes was quite unexpected. The effect of chronic 323 RFRP3 treatment on cumulative food intake in the seasonal hamster is in agreement with previous studies 324 reporting that acute RFRP3 treatment increases food intake in mice, jerboas, rats, ewes and non-human 325 primates ${ }^{20,21,25,42}$. Some studies have proposed that RFRP3 may act directly on NPY and POMC neurons. 326 Thus, in ewe RFRP3 fibers project to NPY and POMC neurons ${ }^{59}$ and acute RFRP3 treatment increases their 327 neuronal activity ${ }^{21}$. Further, in the female jerboas the acute orexigenic effect of RFRP3 is associated with 328 increased Npy mRNA and decreased Pomc mRNA expression ${ }^{25}$ and in male rats, RFRP3 treatment 329 increased food intake and Npy mRNA levels in the ARC ${ }^{21}$. Interestingly, none of these short-term effects 330 of RFRP3 on food intake and gene expression were associated with increased body weight supporting the 331 idea that the chronic (photoperiodic) effects of RFRP3 on body weight may use different metabolic 332 pathways and do not depend on changes in NPY and POMC. Actually, a study reported that Djungarian 333 hamsters over-expressing the orexigenic AgRP, although exhibiting higher food intake and lower energy 334 expenditure relative to the control hamsters, still show a significant, prolonged decrease in body weight 335 when exposed to SD conditions ${ }^{60}$. Alternatively, if leptin increases POMC and decreases NPY expression, 336 as it does in other animals ( ${ }^{61}$ for review) we cannot exclude that RFRP3 affects the neuronal activity of 337 NPY and POMC neurons and counteracts the effects of leptin, resulting in no apparent changes. Finally, 338 the chronic RFRP3 treatment may have caused changes in peptide content or release, which were not 339 evaluated in our study. 
340 Overall, our study shows that the central RF-amide peptides Kp and RFRP3, which are implicated in the 341 control of reproduction, notably in seasonal species, may also participate in the seasonal regulation of 342 body weight, at least in male Djungarian hamsters. Our results indicate that Kp modulates the seasonal 343 changes in body weight in a sex-steroid dependent manner, and through an action on ARC POMC and NPY 344 neurons. The long term effect of RFRP3 on body weight by contrast seems sex-steroid independent and 345 does not appear to involve the ARC POMC and NPY neurons, but possibly a different neuro-endocrine 346 pathway involving the control of food intake and adiposity. Further experiments are now necessary to 347 dissect in more detail how Kp and RFRP3 separately affect lean (through the anabolic effect of 348 testosterone) and fat mass. In conclusion, using a relevant seasonal model our study adds further support 349 to the increasing evidence for an integrated regulation of both metabolism and reproduction.

\section{Acknowledgements}

352 With special thanks to Dr Dominic Ciocca, Aurore Senser and Nicolas Lethenet for the help provided with 353 the caring of the animals used in this study. We also would like to thank to Clarisse Quignon for her 354 technical support. This project has been funded with support from the NeuroTime Erasmus+ program of 355 the European Commission and the Repramide ANR-13-BSV1-001.

\section{References}

360 1. Figala AJ, Hoffmann K, Goldau G. International Association for Ecology Zur Jahresperiodik beim Dsungarischen Zwerghamster Phodopus sungorus Pallas ( The Annual Cycle in the Djungarian 

Association. 1973;12(2):89-118.

364 2. Bartness TJ, Wade GN. Photoperiodic control of seasonal body weight cycles in hamsters. Neurosci Biobehav Rev. 1985;9(4):599-612.

3. Warner A, Jethwa PH, Wyse CA, I'Anson H, Brameld JM, Ebling FJP. Effects of photoperiod on daily locomotor activity, energy expenditure, and feeding behavior in a seasonal mammal. AJP Regul Integr Comp Physiol. 2010;298(5):R1409-R1416.

4. Bartness TJ, Powers JB, Hastings MH, Bittman EL, Goldman BD. The timed infusion paradigm for melatonin delivery: What has it taught us about the melatonin signal, its reception, and the photoperiodic control of seasonal responses? J Pineal Res. 1993;15(4):161-190.

372 5. Gottsch ML, Cunningham MJ, Smith JT, et al. A role for kisspeptins in the regulation of gonadotropin 373 secretion in the mouse. Endocrinology. 2004;145(9):4073-4077.

374 6. Kriegsfeld LJ, Mei DF, Bentley GE, et al. Identification and characterization of a gonadotropininhibitory system in the brains of mammals. Proc Natl Acad Sci U S A. 2006;103(1650):2410-2415.

376 7. Revel FG, Saboureau M, Masson-Pévet M, Pévet P, Mikkelsen JD, Simonneaux V. Kisspeptin Mediates the Photoperiodic Control of Reproduction in Hamsters. Curr Biol. 2006;16:1730-1735.

378 8. Ubuka T, Inoue K, Fukuda Y, et al. Identification, expression, and physiological functions of Siberian hamster gonadotropin-inhibitory hormone. Endocrinology. 2012;153(1):373-385.

9. Revel FG, Saboureau M, Pévet P, Simonneaux V, Mikkelsen JD. RFamide-related peptide gene is a melatonin-driven photoperiodic gene. Endocrinology. 2008;149(3):902-912. central for seasonal reproduction. Curr Biol. 2014;24(13):1500-1506. 
384 11. Simonneaux V. A Kiss to drive rhythms in reproduction. Eur J Neurosci. 2018;(October):0-2.

385 12. Henningsen JB, Gauer F, Simonneaux V. RFRP neurons - the doorway to understanding seasonal reproduction in mammals. Front Endocrinol (Lausanne). 2016;7(MAY):1-10.

13. Ancel C, Bentsen AH, Sébert ME, Tena-Sempere M, Mikkelsen JD, Simonneaux V. Stimulatory effect of RFRP-3 on the gonadotrophic axis in the male Syrian hamster: The exception proves the rule. Endocrinology. 2012;153(January):1352-1363.

14. Rasri-Klosen K, Simonneaux V, Klosen P. Differential response patterns of kisspeptin and RFRP to photoperiod and sex steroid feedback in the Djungarian hamster (Phodopus sungorus). J Neuroendocrinol. 2017;3(May):1-13.

15. Mercer JG, Moar KM, Ross a W, Hoggard N, Morgan PJ. Photoperiod regulates arcuate nucleus POMC, AGRP, and leptin receptor mRNA in Siberian hamster hypothalamus. Am J Physiol Regul Integr Comp Physiol. 2000;278(14):R271-R281.

16. Herwig a., Petri I, Barrett P. Hypothalamic Gene Expression Rapidly Changes in Response to Photoperiod in Juvenile Siberian Hamsters (Phodopus sungorus). J Neuroendocrinol. 2012;24(4):991-998.

17. Tolson KP, Garcia C, Yen S, et al. Brief report Impaired kisspeptin signaling decreases metabolism 400 and promotes glucose intolerance and obesity. 2014;124(7):3075-3079.

18. De Bond JAP, Tolson KP, Nasamran C, Kauffman AS, Smith JT. Unaltered Hypothalamic Metabolic Gene Expression in Kiss1r Knockout Mice Despite Obesity and Reduced Energy Expenditure. J Neuroendocrinol. 2016;28(10):1-10.

19. León S, García-Galiano D, Ruiz-Pino F, et al. Physiological roles of gonadotropin-inhibitory hormone 405 signaling in the control of mammalian reproductive axis: Studies in the NPFF1 receptor null mouse. 
20. Johnson MA, Tsutsui K, Fraley GS. Rat RFamide-related peptide-3 stimulates GH secretion , inhibits LH secretion, and has variable effects on sex behavior in the adult male rat. 2007;51:171-180.

21. Clarke IJ, Smith JT, Henry BA, et al. Gonadotropin-inhibitory hormone is a hypothalamic peptide that provides a molecular switch between reproduction and feeding. Neuroendocrinology.

22. Klosen P, Maessen X, van den Bosch de Aguilar P. PEG embedding for immunocytochemistry: application to the analysis of immunoreactivity loss during histological processing. $J$ Histochem Cytochem. 1993;41(3):455-463.

23. Klosen P, Sébert ME, Rasri K, Laran-Chich MP, Simonneaux V. TSH restores a summer phenotype in photoinhibited mammals via the RF-amides RFRP3 and kisspeptin. FASEB J. 2013;27(7):2677-2686.

24. Rasri K, Mason P, Govitrapong P, Pevet P, Klosen P. Testosterone-driven seasonal regulation of vasopressin and galanin in the bed nucleus of the stria terminalis of the Djungarian hamster (Phodopus sungorus). Neuroscience. 2008;157(1):174-187.

420 25. Talbi R, Laran-Chich M-P, Magoul R, El Ouezzani S, Simonneaux V. Kisspeptin and RFRP-3 421 differentially regulate food intake and metabolic neuropeptides in the female desert jerboa. Sci Rep. 2016;6(1):36057.

26. Dudek M, Ziarniak K, Sliwowska JH. Kisspeptin and metabolism: The brain and beyond. Front Endocrinol (Lausanne). 2018;9(APR):1-8.

27. Schneider JE, Benton NA, Russo KA, et al. RFamide-related peptide-3 and the trade-off between reproductive and ingestive behavior. Integr Comp Biol. 2017;57(6):1225-1239. 
of metabolic and reproductive hypothalamic peptides in the desert jerboa. J Comp Neurol. 2016;524(18):3717-3728.

430

29. Garcia NW, Greives TJ, Zysling D a, French SS, Chester EM, Demas GE. Exogenous insulin enhances humoural immune responses in short-day, but not long-day, Siberian hamsters (Phodopus sungorus). Proc Biol Sci. 2010;277(March):2211-2218.

30. Klingenspor M, Niggemann H, Heldmaier G. Modulation of leptin sensitivity by short photoperiod acclimation in the Djungarian hamster, Phodopus sungorus. J Comp Physiol B. 2000;170:37-43.

31. Adam CL, Moar KM, Logie TJ, et al. Photoperiod Regulates Growth, Puberty and Hypothalamic Neuropeptide and Receptor Gene Expression in Female Siberian Hamsters ${ }^{1}$. Endocrinology. 2000;141(12):4349-4356.

32. Mason AO, Greives TJ, Scotti MAL, et al. Suppression of kisspeptin expression and gonadotropic axis sensitivity following exposure to inhibitory day lengths in female Siberian hamsters. Horm Behav. 2007;52(4):492-498.

33. Thompson EL, Patterson M, Murphy KG, et al. Central and peripheral administration of kisspeptin10 stimulates the hypothalamic-pituitary-gonadal axis. J Neuroendocrinol. 2004;16(10):850-858.

34. Greives TJ, Long KL, Bergeon Burns CM, Demas GE. Response to exogenous kisspeptin varies according to sex and reproductive condition in Siberian hamsters (Phodopus sungorus). Gen Comp Endocrinol. 2011;170(1):172-179.

35. Navarro VM, Castellano JM, Fernández-Fernández R, et al. Effects of KiSS-1 peptide, the natural ligand of GPR54, on follicle-stimulating hormone secretion in the rat. Endocrinology. 2005;146(4):1689-1697.

36. Li SN, Xue HL, Zhang Q, et al. Photoperiod regulates the differential expression of KISS-1 and GPR54 
451 37. Revel FG, Masson-Pévet M, Pévet P, Mikkelsen JD, Simonneaux V. Melatonin controls seasonal breeding by a network of hypothalamic targets. Neuroendocrinology. 2009;90(1):1-14.

453 38. Murphy M, Ebling FJP. The role of hypothalamic tri-iodothyronine availability in seasonal regulation 454 of energy balance and body weight. J Thyroid Res. 2011;2011.

39. Henson JR, Carter SN, Freeman DA. Exogenous T3elicits long day-like alterations in testis size and the RFamides kisspeptin and gonadotropin-inhibitory hormone in short-day Siberian hamsters. J Biol Rhythms. 2013;28(3):193-200.

40. Paul MJ, Pyter LM, Freeman DA, Galang J, Prendergast BJ. Photic and nonphotic seasonal cues differentially engage hypothalamic kisspeptin and RFamide-related peptide mRNA expression in Siberian hamsters. J Neuroendocrinol. 2009;21(12):1007-1014.

41. Wade GN, Bartness TJ, Wade N. Effects of photoperiod and gonadectomy on food intake, body weight, and body composition in Siberian hamsters Effects of photoperiod and gonadectomy on food intake, body weight, and body composition in Siberian hamsters. Am J Physiol Regul Integr Comp Physiol. 1984;256:R26-R30.

42. Anjum S, Krishna A, Tsutsui K. Possible role of GnIH as a mediator between adiposity and impaired testicular function. Front Endocrinol (Lausanne). 2016;7(FEB):1-12.

43. Ye J. Mechanisms of insulin resistance in obesity. Front Med. 2013;7(1):14-24.

44. Tups A, Ellis C, Moar KM, et al. Photoperiodic Regulation of Leptin Sensitivity in the Siberian Hamster, Phodopus sungorus, Is Reflected in Arcuate Nucleus SOCS-3 (Suppressor of Cytokine Signaling) Gene Expression. Endocrinology. 2004;145(3):1185-1193.

45. Tups A, Helwig M, Stöhr S, Barrett P, Mercer JG, Klingenspor M. Photoperiodic regulation of insulin 
receptor mRNA and intracellular insulin signaling in the arcuate nucleus of the Siberian hamster,

473 Phodopus sungorus. Am J Physiol Regul Integr Comp Physiol. 2006;291(3):R643-50.

474 46. Higo S, Honda S, lijima N, Ozawa H. Mapping of Kisspeptin Receptor mRNA in the Whole Rat Brain 475 and its Co-Localisation with Oxytocin in the Paraventricular Nucleus. J Neuroendocrinol. $476 \quad 2016 ; 28(4): 1-8$.

477 47. Henningsen JB, Poirel VJ, Mikkelsen JD, Tsutsui K, Simonneaux V, Gauer F. Sex differences in the 478 photoperiodic regulation of RF-Amide related peptide (RFRP) and its receptor GPR147 in the syrian 479 hamster. J Comp Neurol. 2016;524(9):1825-1838.

48. Higo S, lijima N, Ozawa H. Characterisation of Kiss1r ( Gpr54 )-Expressing Neurones in the Arcuate 481 Nucleus of the Female Rat Hypothalamus. J Neuroendocrinol. 2017;29(2):1-8.

49. Fu L-Y, van den Pol AN. Kisspeptin Directly Excites Anorexigenic Proopiomelanocortin Neurons but Inhibits Orexigenic Neuropeptide Y Cells by an Indirect Synaptic Mechanism. J Neurosci.

50. Chowen-Breed J, Fraser HM, Vician L, Damassa DA, Clifton DK, Steiner RA. Testosterone regulation of proopiomelanocortin messenger ribonucleic acid in the arcuate nucleus of the male rat.

51. Adams LA, Vician L, Clifton DK, Steiner RA. Testosterone Regulates Proopiomelanocortin GeneExpression in the Primate Brain. Endocrinology. 1991;128(4):1881-1886.

52. Hileman SM, Lubbers LS, Petersen SL, KuehI DE, Scott CJ, Jackson GL. Influence of testosterone on LHRH release, LHRH mRNA and proopiomelanocortin mRNA in male sheep. J Neuroendocrinol. 1996;8(2):113-121. 

selected hypothalamic sites and in vitro release from the medial basal hypothalamus of castrated male rats. Endocrinology. 1989;124(1):410-414.

54. Urban JH, Bauer-Dantoin A C., Levine JE. Neuropeptide Y Gene Expression in the Arcuate Nucleus: Sexual Dimorphism and Modulation by Testosterone. 1993;132(1):139-145.

55. Sohn EH, Wolden-Hanson T, Matsumoto AM. Testosterone (T)-induced changes in arcuate nucleus cocaine-amphetamine-regulated transcript and NPY mRNA are attenuated in old compared to young male Brown Norway rats: Contribution of T to age-related changes in cocaine-amphetamineregulated transcript . Endocrinology. 2002;143(3):954-963.

56. Helwig $\mathrm{H}$, Khorooshi RMH, Tups $\mathrm{A}$, et al. PC1/3 and PC2 gene expression and post-translational 503

59. Qi Y, Oldfield BJ, Clarke IJ. Projections of RFamide-related peptide-3 neurones in the ovine

58. Nestor CC, Qiu J, Padilla SL, et al. Optogenetic Stimulation of Arcuate Nucleus Kiss1 Neurons Reveals a Steroid-Dependent Glutamatergic Input to POMC and AgRP Neurons in Male Mice. Mol Endocrinol. 2016;30(6):630-644. overexpression of the agouti-related peptide gene. J Neuroendocrinol. 2010;22(6):564-575. 
516 61. Varela L, Horvath TL. Leptin and insulin pathways in POMC and AgRP neurons that modulate energy 517 balance and glucose homeostasis. EMBO Rep. 2012;13(12):1079-1086. 
520 Figure 1. Dose dependent effect of RFRP3 in male and female Djungarian hamsters. Male (A) or female (B)

521 hamsters were raised in conditions of long days (LD, white bars) or short days (SD, grey bars) and treated

522 with a 5 week-intra-cerebro-ventricular infusion of artificial cerebrospinal fluid (aCSF) or various

523 concentrations (from) RFRP3. At the end of treatment, paired testicles and seminal vesicles weight in male

524 and uterus weight in female were measured. The body weight change was calculated for both male and

525 female during the 5 week treatment. Data presented are mean \pm SEM of $n=6$ to 7 per experimental group,

526 and scattered dots represent individual data -. * $\mathrm{P}<0.05$; after One-way ANOVA and Tukey's post hoc

527 honestly significance test, different vs SD aCSF group.

528 Figure 2. Effect of kisspeptin or RFRP3 on the reproductive status of photoinhibited male and female

529 Djungarian hamsters. Male (A,B) or female (C) hamsters were raised in long day (LD) or short day (SD)

530 conditions and treated with a 5 week intracerebroventricular (icv) infusion of artificial cerebrospinal fluid

531 (aCSF), kisspeptin 10 (Kp10, 170 pmol/hour) or RFRP3 (8.25 pmol/hour). Testes and seminal vesicle values

532 are given as paired weight. Data represented in bars are mean \pm SEM of $n=5$ to 7 per experimental group,

533 and the dots represent individual data values. Different letters mean the groups are statistically different

534 after One-way ANOVA and Tukey's post hoc honestly significance test.

535 Figure 3. Effect of kisspeptin or RFRP3 on the metabolic status of photoinhibited male and female

536 Djungarian hamsters. At week 0, male (A) or female (B) hamsters raised in long days (LD) were either kept

537 in LD (grey line) or placed in short day (SD) conditions (black lines). After 10 weeks (vertical dotted line)

538 hamsters were treated with a 5 week intracerebroventricular (icv) infusion of artificial cerebrospinal fluid

539 (aCSF), $0.17 \mathrm{nmol} /$ hour kisspeptin 10 (Kp10) or $8.25 \mathrm{pmol} /$ hour RFRP3. The body weight (BW) was followed

540 weekly since the beginning of the experiment (week 0). Food intake was monitored once a week since the

541 start of the ICV infusion (Week 10). The change (delta) in BW and cumulative food intake are summed for 
542 the 5 weeks of treatment. Plasma levels of glucose, insulin and leptin were measured at the end of the 5

543 week treatment. Data are presented as mean \pm SEM of $n=6$ to 7 per experimental group and the dots

544 represent individual data values. Different letters in histograms mean the groups are statistically different

545 after One-way ANOVA and Tukey's post hoc honestly significance test.

546 Figure 4. Effect of kisspeptin or RFRP3 on metabolic gene expression in the arcuate nucleus of 547 photoinhibited male Djungarian hamsters. Male hamsters were raised in of long day (LD) or short day (SD) 548 conditions and treated with a 5 week intracerebroventricular infusion of artificial cerebrospinal fluid (aCSF), $549170 \mathrm{pmol} /$ hour kisspeptin 10 (Kp10) or $8.25 \mathrm{pmol} / \mathrm{hour}$ RFRP3. At the end of the treatment, in situ 550 hybridization was performed for gene encoding A - POMC (pro-opiomelanocortin), B - NPY (neuropeptide 551 Y) and C - SST (somatostatin). Pictures show representative images of the arcuate neurons expressing 552 Pomc, Npy and Sst mRNA after the different treatments (scale bar=100 $\mu \mathrm{m}$ ). Bars show the semi553 quantification of the mean number of labeled neurons per animal (left histogram) or the mean labeling 554 intensity (in arbitrary units) per neuron (right histogram). Data are presented as mean \pm SEM of $n=6$ to 7 555 per experimental group, and the dots represent individual data values. Different letters mean the groups 556 are statistically different after One-way ANOVA and Tukey's post hoc honestly significance test.

557 Figure 5. Effect of kisspeptin or RFRP3 on metabolic gene expression in the arcuate nucleus of 558 photoinhibited female Djungarian hamsters. Female hamsters were raised in of long day (LD) or short day 559 (SD) conditions and treated with a 5 week intracerebroventricular infusion of artificial cerebrospinal fluid 560 (aCSF), $170 \mathrm{pmol} /$ hour kisspeptin 10 (Kp10) or $8.25 \mathrm{pmol} / \mathrm{hour} \mathrm{RFRP3.} \mathrm{At} \mathrm{the} \mathrm{end} \mathrm{of} \mathrm{the} \mathrm{treatment,} \mathrm{in} \mathrm{situ}$ 561 hybridization was performed for gene encoding A - POMC (pro-opiomelanocortin), B - NPY (neuropeptide 562 Y) and C - SST (somatostatin). Pictures show representative images of the arcuate neurons expressing 563 Pomc, Npy and Sst mRNA after the different treatments (scale bar=100 $\mu \mathrm{m}$ ). Bars show the semi564 quantification of the mean number of labeled neurons per animal (left histogram) or the mean labeling 565 intensity (in arbitrary units) per neuron (right histogram). Data are presented as mean \pm SEM of $n=6$ to 7 
566 per experimental group, and the dots represent individual data values. Different letters mean the groups

567 are statistically different after One-way ANOVA and Tukey's post hoc honestly significance test.

568 Figure 6. Effect of castration on reproductive and metabolic physiology in kisspeptin-treated male 569 Djungarian hamster. Hamsters adapted to the inhibitory short day (SD) conditions were gonadectomized 570 (Gx, open symbols) or sham operated (sham, closed symbols), then were treated with a 5 week 571 intracerebroventricular infusion of artificial cerebrospinal fluid (aCSF) or 170 pmol/hour kisspeptin. At the 572 end of the treatment, A- the reproductive function was evaluated with the paired seminal vesicle weight, 573 and the metabolism was measured by B - the change in body weight (BW) and C - the cumulative food 574 intake. Data are presented as mean \pm SEM. of $n=6$ to 7 per experimental group and the points represent 575 individual data values. * $\mathrm{P}<0.05$ after Two-way ANOVA and Tukey's post hoc honestly significance test vs 576 Sham + aCSF. 
Testicles

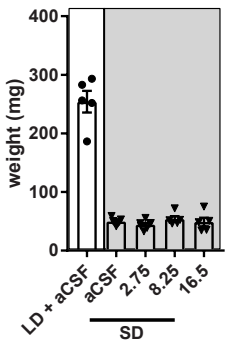

RFRP3 dose ( $\rho \mathrm{mol} /$ hour)
Seminal Vesicles

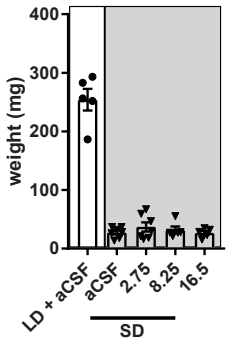

RFRP3 dose ( $\rho \mathrm{mol} / \mathrm{hour}$ )
BW change

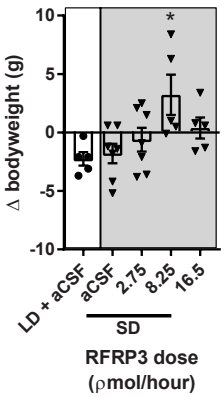

Females

\section{Uterus}

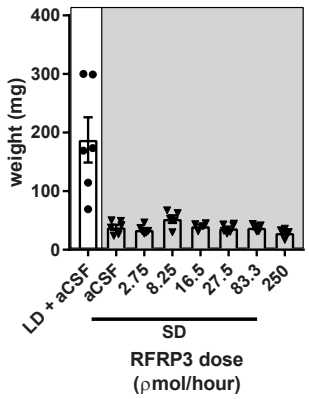

BW change

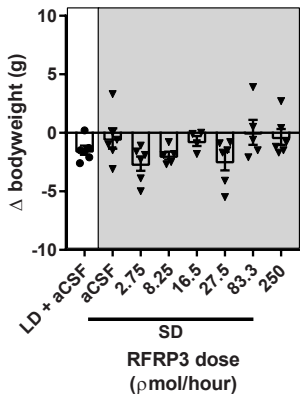


Testicles

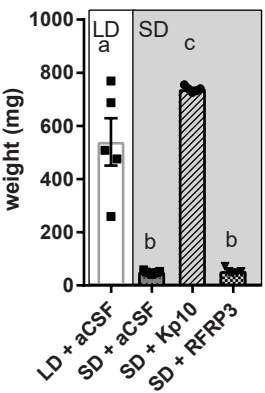

Seminal Vesicles

\section{Uterus}

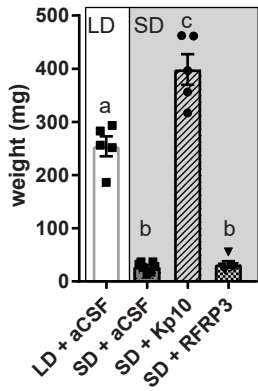


A

LD + aCSF

SD + aCSF

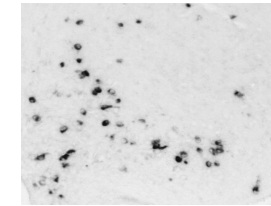

SD + Kp10

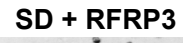

Pomc neurons

Pomc intensity

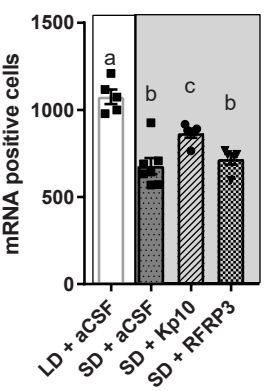

B
LD + aCSF
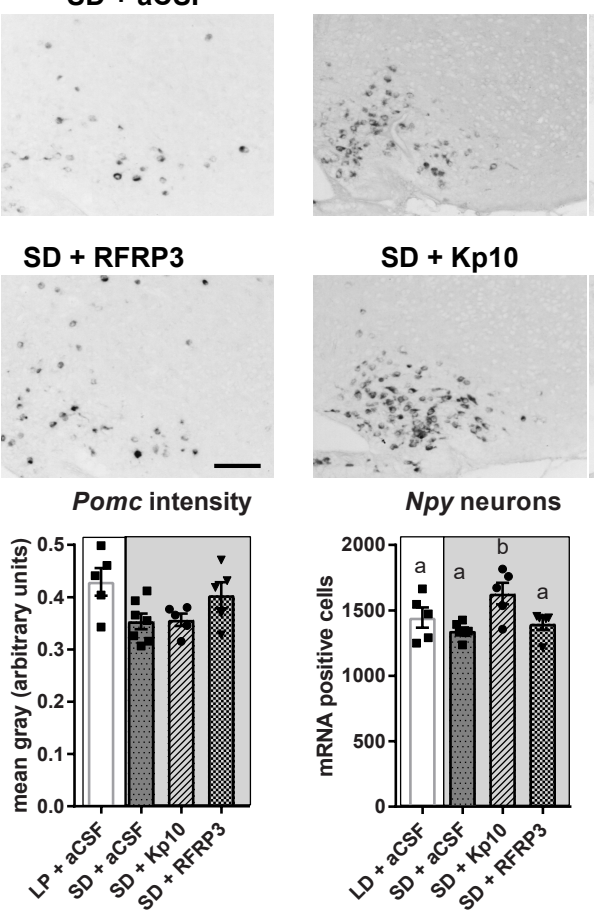

SD + Kp10

Npy neurons

$S D+a C S F$

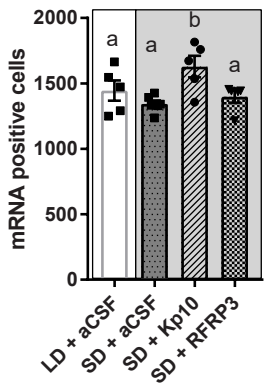

SD + RFRP3
C

\section{LD + aCSF}

$S D+a C S F$
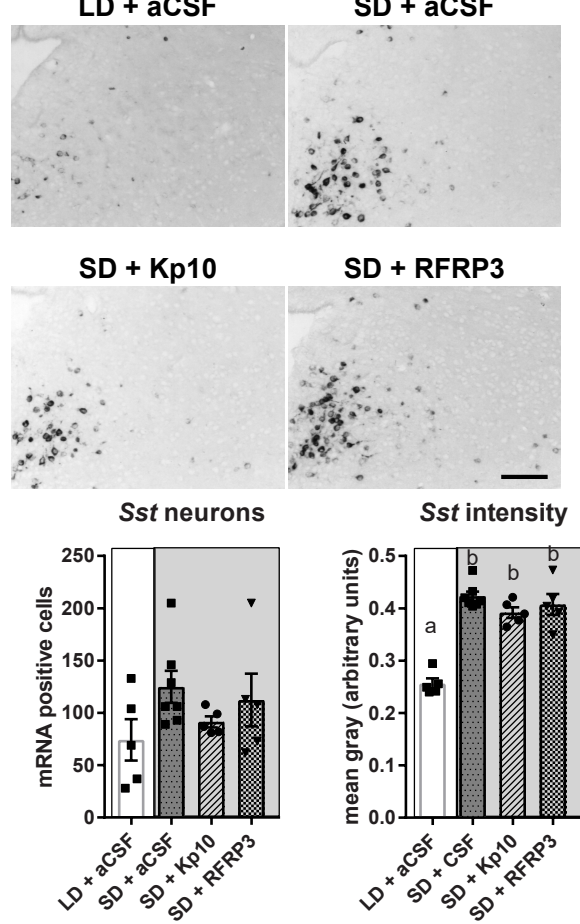

SD + RFRP3

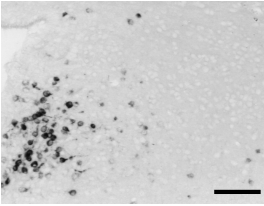

Sst intensity
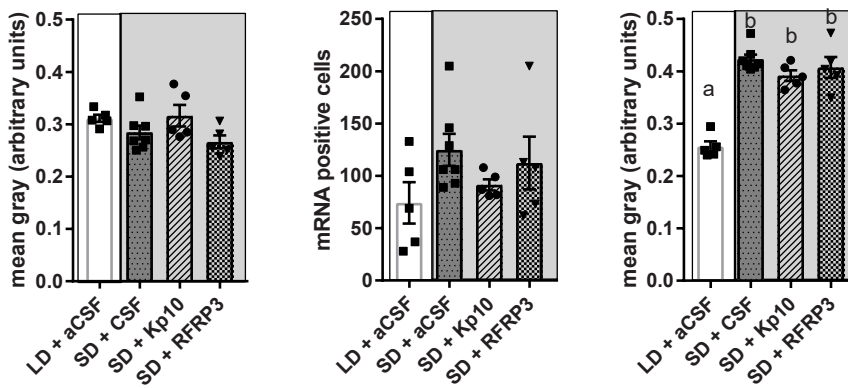


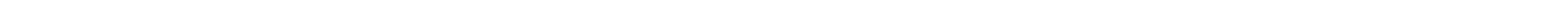




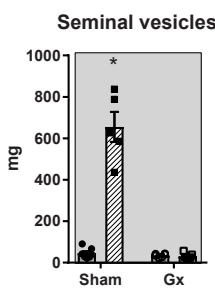

BW change

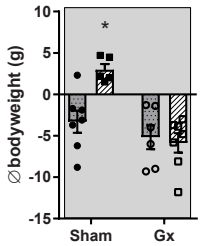

Cumulative Food intake

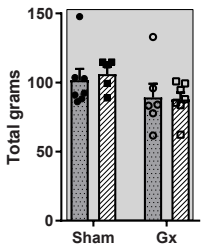

四 aCSF Im Kp10 